\title{
Dosimetric Evaluation of Heterogeneities in Small Circular Fields of 6 MV Photon Beams with EBT2 and EDR2 Films: Comparison with Monte Carlo Calculation
}

\author{
Ali Mohammad' ${ }^{1}$ Hassan A. Nedaie ${ }^{1 *}$, Mehran YarAhmadi'2, Nooshin Banaee ${ }^{3}$, \\ Mansour Naderi ${ }^{1}$, Zohre Tizmaghz ${ }^{1}$ \\ ${ }^{1}$ Radiotherapy Oncology Department, Cancer Research Centre, Cancer Institute, Tehran University of Medical \\ Sciences, Tehran, Iran \\ ${ }^{2}$ Department of Medical Physics, Faculty of Medicine, Kurdistan University of Medical Sciences, Sanandaj, Iran \\ ${ }^{3}$ Young Researchers and Elites Club, Science and Research Branch, Islamic Azad University, Tehran, Iran \\ Email: ${ }^{*}$ Nedaieha@sina.tums.ac.ir
}

Received 2 August 2014; revised 28 August 2014; accepted 15 September 2014

Copyright (C) 2014 by authors and Scientific Research Publishing Inc.

This work is licensed under the Creative Commons Attribution International License (CC BY). http://creativecommons.org/licenses/by/4.0/

(c) (i) Open Access

\section{Abstract}

The influence of dose variation due to heterogeneities in narrow photon beams used in stereotactic radiosurgery has been investigated. Since the lateral electronic disequilibrium and existence of steep dose gradients in small fields and the presence of heterogeneities can intensify these problems, in this study the effects of heterogeneities on $6 \mathrm{MV}$ small photon beams produced by circular cone collimators with 5, 10, 15, 20 and $30 \mathrm{~mm}$ diameters are investigated. The heterogeneities include $3 \mathrm{~cm}$ Cork with density of $0.2 \mathrm{~g} / \mathrm{cm}^{3}$ instead of lung and $3 \mathrm{~cm}$ Polytetrafluoroethylene (P.T.F.E) with density of $2.2 \mathrm{~g} / \mathrm{cm}^{3}$ as bone. The measurements were carried out with EBT2 gafchromic and EDR2 radiographic films. Simulation was done by MCNP Monte Carlo Code (MCNP5). The depth dose curves in heterogeneous phantom were compared with homogeneous phantom. A good agreement was obtained within film and Monte Carlo calculations in presence of low density heterogeneity and also in the presence of high density heterogeneity. Monte Carlo results showed good agreement after stopping power correction.

\section{Keywords}

Stereotactic Radiosurgery, Heterogeneities, Film Dosimetry, Monte Carlo Calculation

\footnotetext{
${ }^{*}$ Corresponding author.
}

How to cite this paper: Mohammad, A., Nedaie, H.A., YarAhmadi, M., Banaee, N., Naderi, M. and Tizmaghz, Z. (2014) Dosimetric Evaluation of Heterogeneities in Small Circular Fields of 6 MV Photon Beams with EBT2 and EDR2 Films: Comparison with Monte Carlo Calculation. Journal of Modern Physics, 5, 1608-1616. http://dx.doi.org/10.4236/jmp.2014.516162 


\section{Introduction}

In recent years, treatment by linear accelerator-based stereotactic radiosurgery (SRS) has increased significantly. This type of treatment modality employs narrowly-collimated, 6 - 10 radiation photon beams with field sizes smaller than $4 \times 4 \mathrm{~cm}^{2}$ to limited volume of tumor. The aim of this technique is to deliver high dose with accurate position and dose of $\pm 1 \mathrm{~mm}$ of $\pm 5 \%$, respectively [1]. To achive this accuracy, dosimetric measurements are of great importance. Because of the lateral electronic disequilibrium and steep dose gradient existence in large portion of these fields, dosimetry is more complicated [2]. Several studies have surveyed the dosimetry of narrow photon beams in a homogeneous medium [3]-[8] but the effect of density differences on such small beams has not been extensively studied [9]-[14]. In radiotherapy two types of tissue interface are important: low density heterogeneity/soft tissue, bone/soft tissue. The impact of heterogeneities may create perturbation in the dose distribution which can be deceptive in the exact localization of dose ranges. Clinically this may lead to the underdosing of the tumor and overdosing of the normal tissues. Many algorithms such as Batho and equivalent pathlenghth (EPL) avoid dealing with the complexity of charged particle transport (CPT) [15]. That is very important factor to estimate dose in regions without charged particle equilibrium (CPE) such as small fields of SRS in which heterogeneities can change electronic equilibrium. The majority of SRS treatment planning systems ignore the presence of heterogeneities due to computation time limitations and also assumption that multiple beam arrangements are compensating the perturbation effect. Because of the effect of heterogeneities on inaccuracy of the measurements, especially in small fields, selecting detectors with good spatial resolution, high accuracy and precision, dose rate and energy independence is very important. Although ionization chamber has good accuracy in measuring of absolute dose but in regions with steep dose gradient and charged particle disequilibrium, volume of detector affects on accuracy of the measurements [16]. Diode detectors are similar to ion chambers but their response is directionally dependant. TLD dosimeters also would require a large number of them to provide adequate information. Film is two-dimensional dosimeter with good spatial resolution for surveying pixel-by-pixel of measurements. Monte Carlo method provides a bridge between measurement and calculation and it is one of the best methods for situations in which physical measurements are difficult or impossible.

The aim of this study is to evaluate the effects of heterogeneities (low and high densities) of SRS fields irradiated by $6 \mathrm{MV}$ photon beams using EBT2 and EDR2 films and also verification by Monte Carlo simulation.

\section{Materials and Methods}

\subsection{Phantoms}

To evaluate dose perturbation caused by heterogeneities, PMMA slab phantom with $3 \mathrm{~cm}$ thickness of cork and density of $0.2 \mathrm{~g} / \mathrm{cm}^{3}$ (low density heterogeneity) and $3 \mathrm{~cm}$ thickness of Polytetrafluoroethylene (P.T.F.E) with density of $2.2 \mathrm{~g} / \mathrm{cm}^{3}$ (high density heterogeneity) were applied as lung and bone, respectively.

\subsection{Kodak EDR2 Film}

The radiographic film type EDR2 (Kodak Extended Dose Range-2, Rochester, New York 14650) is a very slow speed and fine-grain film. Double emulsion active layers and $2.3 \mathrm{~g} / \mathrm{m}^{2}$ of cubic microcrystal AgBr grains with density of $6.53 \mathrm{~g} / \mathrm{cm}^{3}$ (average size of 0.24 micrometer) coated on $0.18 \mathrm{~mm}$ Easter base. EDR2 film is less sensitive to processor variations than XV2 film that allows processing of films in a routine film processor [17]. In order to prevent film batch differences, all films (with size of $35 \times 43 \mathrm{~cm}^{2}$ ) were chosen from the same batch [18].

\subsection{Radiochromic Film}

EBT2 Gafchromic films (International Specialty Products) were used as an alternative dosimeter with the composition of $40.85 \% \mathrm{H}, 0.10 \% \mathrm{Li}, 42.37 \% \mathrm{C}, 0.01 \% \mathrm{~N}, 16.59 \% \mathrm{O}, 0.04 \% \mathrm{Cl}, 0.01 \% \mathrm{~K}$ and $0.01 \% \mathrm{Br}$, with effective atomic number of 6.84, as reported by manufacturer. EBT2 contains a yellow "marker" dye, which minimizes response differences caused by coating anomalies. EBT2 is nearly 10× less sensitive to cool white fluorescent light than EBT films. The spectral absorbance peak of EBT2 is about $636 \mathrm{~nm}$. According to the recommendation of the manufactures, all films should be scanned in landscape mode and red channel, because the 
yellow marker dye has no absorbance in the red portion of the spectrum and therefore has no effect on the film response in the red color channel. All films (size of $20 \times 25 \mathrm{~cm}^{2}$ ) were chosen from the same batch.

\subsection{Monte Carlo Simulation}

The simulation was done by MCNP5 Monte Carlo (MC) radiation transport code (Los Alamos National Laboratory). The MCPLIB04 photon cross-section library was applied using data from ENDF/B-VI. The results from the MCNP5 calculations contain pulse height tally for energy or charge deposition, to calculate relative dose in this study.

\subsection{Dose Delivery}

The Films were irradiated by 6 MV photon beams produced by Clinac 2100C/D (Varian Medical Systems, Inc., Palo Alto, CA) which were collimated by circular cone collimators with sizes of 5, 10, 15, 20, $30 \mathrm{~mm}$ diameters.

\subsection{Film Calibration}

To acquire the relationship between the optical density of the EDR2 film and absorbed dose, The EDR2 film was placed perpendicular to the central beam axis under $5 \mathrm{~cm}$ of polystyrene with $15 \mathrm{~cm}$ of polystyrene underneath the film. The gantry and the collimator angles were $0^{\circ}$ and the source-to-surface distance (SSD) was 100 $\mathrm{cm}$. Eight different dose levels (25, 50, 75, 100, 125, 150, 200, $250 \mathrm{cGy})$ were delivered with a field size of $5 \times$ $5 \mathrm{~cm}^{2}$. An unexposed film was left to determine the optical density (OD) corresponding to the base and fog in such a calibration set (Figure 1(a)). For calibration of EBT2 film, the film was cut into 30 pieces with size of 2 $\times 3 \mathrm{~cm}^{2}$, and the films were placed perpendicular to the central beam axis under $2 \mathrm{~cm}$ of polystyrene with $15 \mathrm{~cm}$ of polystyrene underneath the film, similar to EDR2 setup (Figure 1(b)). For each dose level, three pieces of films were chosen from different portions of original film. The irradiated films were scanned in RGB (Red, Green, Blue) mode and landscape direction 24 hours after irradiation. Before scanning the films, MICROTEK 9800 XL scanner was calibrated with Q-60E calibration strip.

\subsection{Measurement and Calculation}

The shape of rectangular slab phantom is shown in Figure 2. For measurements, each film was packed tightly in the center of the phantom and the radiation beams were impinging parallel to the film. SSD was $100 \mathrm{~cm}$ and 100 MU was applied for each radiation beam at all irradiation steps of this study. In order to verify the simulations, calculated PDD and beam profiles of $10 \times 10 \mathrm{~cm}^{2}$ field size were compared with those obtained by ionization chamber measurements. The same setup was repeated for circular cones as well. To reduce run time, electron and photon energy cut off were set to 0.5 and $0.01 \mathrm{MeV}$, respectively. Also one phase space file with histories of 3 billions was acquired after the flattening filter (the place that was constant for all of configurations). The dose

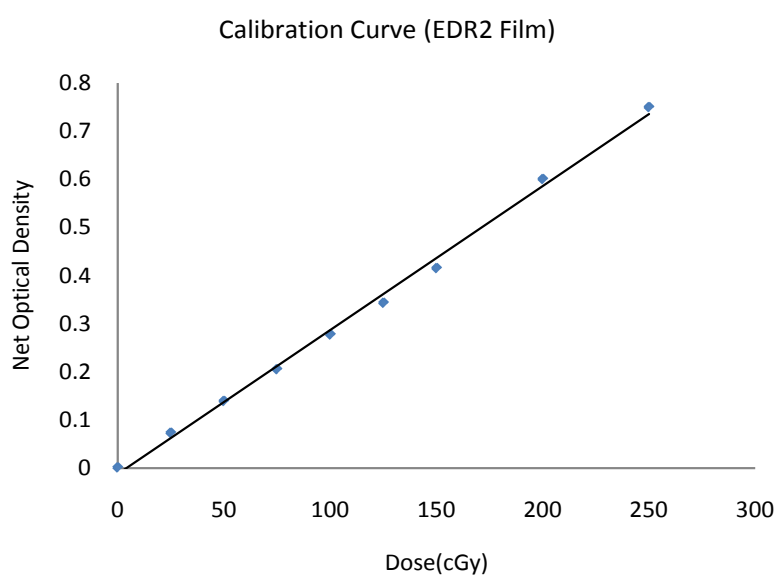

(a)

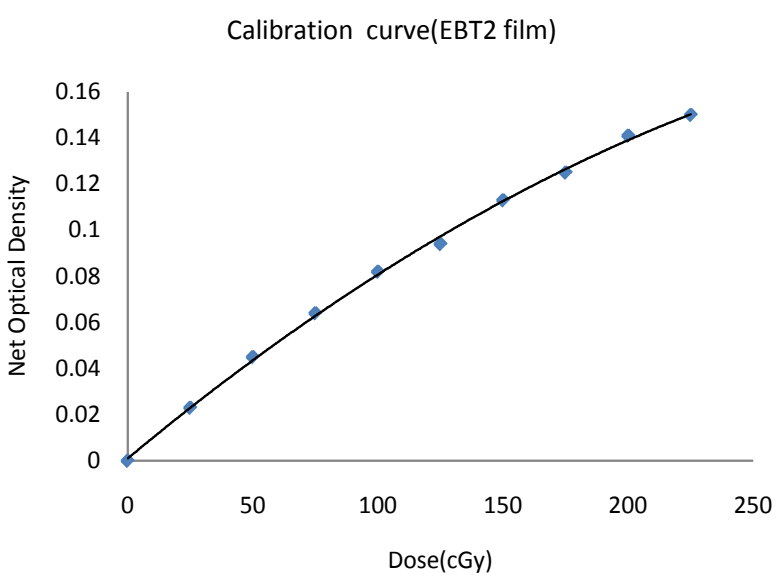

(b)

Figure 1. Calibration curves for (a) EDR2, (b) EBT2 films. 


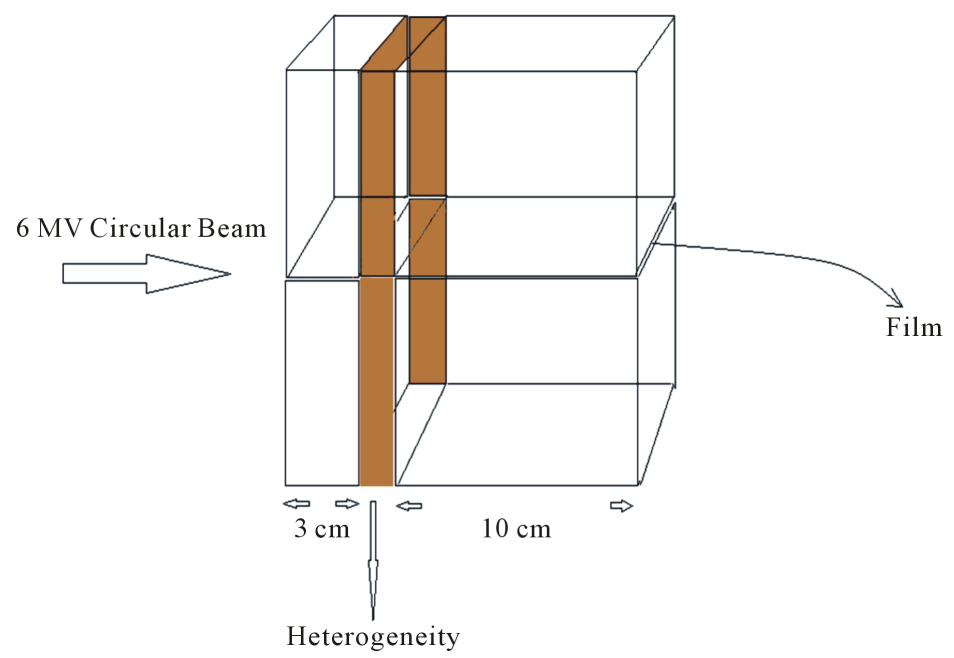

Figure 2. Setup of examination contains rectangular slabs of polyethylene (soft tissue), $3 \mathrm{~cm}$ cork (low density heterogeneity) and $3 \mathrm{~cm}$ P.T.F.E ( high density heterogeneity).

voxels were different for each collimator sizes. The accepted statistical uncertainty was below $2 \%$ for all simulations.

\section{Results and Discussion}

\subsection{Low Density Heterogeneity}

The measured depth doses with EBT2 and EDR2 films in heterogeneous phantom included $3 \mathrm{~cm}$ cork along with those calculated by MCNP5 code are shown in Figure 3(a)-(d). Maximum dose variations for each field size are listed in Table 1. The results show dose reduction at central axis by depth increasing, this reduction is higher for smaller field sizes. Re-buildup region after the heterogeneity almost is independent of field size.

\subsection{High Density Heterogeneity}

Like the low density heterogeneity, the measured and calculated data of heterogeneous phantom consisting of 3 $\mathrm{cm}$ P.T.F.E are shown in Figure 4. Figure 5 illustrates the MCNP5 data after correction and also comparison between perturbations caused by heterogeneity. Maximum dose variations for each field size are tabulated in Table 2. As it can be seen (Figure 4), dose increases at the first layer on central axis, while MCNP5 shows dose increasing in central axis for 5, $10 \mathrm{~mm}$ collimator sizes and decreasing for 15, 20 and $30 \mathrm{~mm}$ collimator sizes.

As it was shown in Figure 4, in the presence of low density heterogeneity, significant perturbation can be observed inside the Cork that). For small field sizes surveyed in this study (5, 10, 15, 20 and $30 \mathrm{~mm}$ ), lateral electronic disequilibrium is very noteworthy and this parameter increases inside the low density heterogeneity. Through the low density of Cork, secondary electrons that leave the field area will increase in comparison to water-equivalent media and this is more important parameter interns if forward range. Increasing the lateral scattering of secondary electrons causes dose reduction in central axis. Although the electron stopping power varies with logarithm of the mean excitation potential of each material, but the average of water-to-lung stopping power ratio is almost 1 [19] and this is consistence with results obtained in this study by Monte Carlo, EBT2 and EDR2 films inside the low density medium. Therefore dose variations in low density heterogeneity depend on density of cork. Also extent of decrease in dose at central axis depends on the field size of radiation beams. With decreasing field size, secondary electrons that leave the field area will increase and this will result in increasing downfall of central axis dose. After the second lung-tissue interface re-buildup is occurred due to decreased attenuation of photon in low density heterogeneity and consequently dose increases proportion to homogeneous phantom. For high density heterogeneity situation is the reverse. Both films showed increase at first layer of P.T.F.E because the films are different in physical density and electron density with bone. Film densities are near the soft tissue therefore, they show dose in unit density of films. Secondary electrons are also 


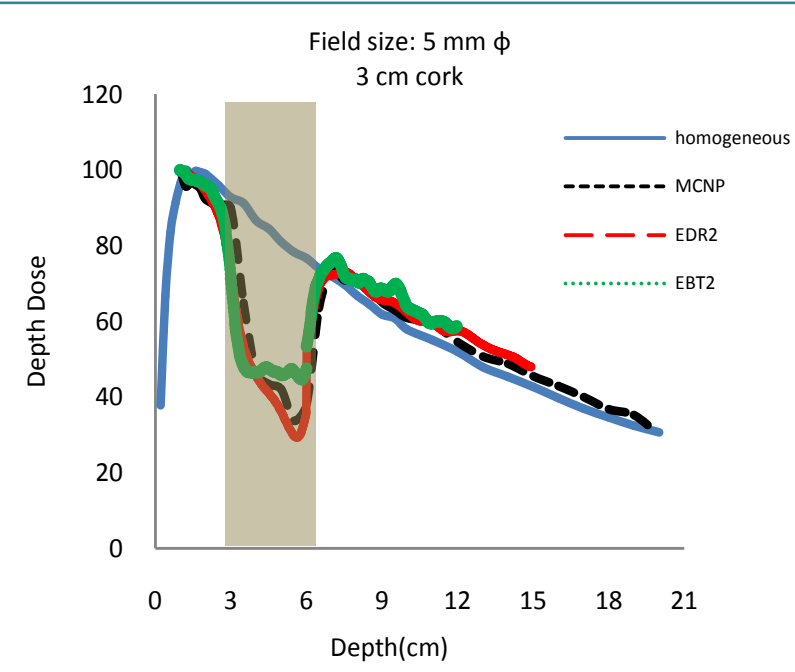

(a)

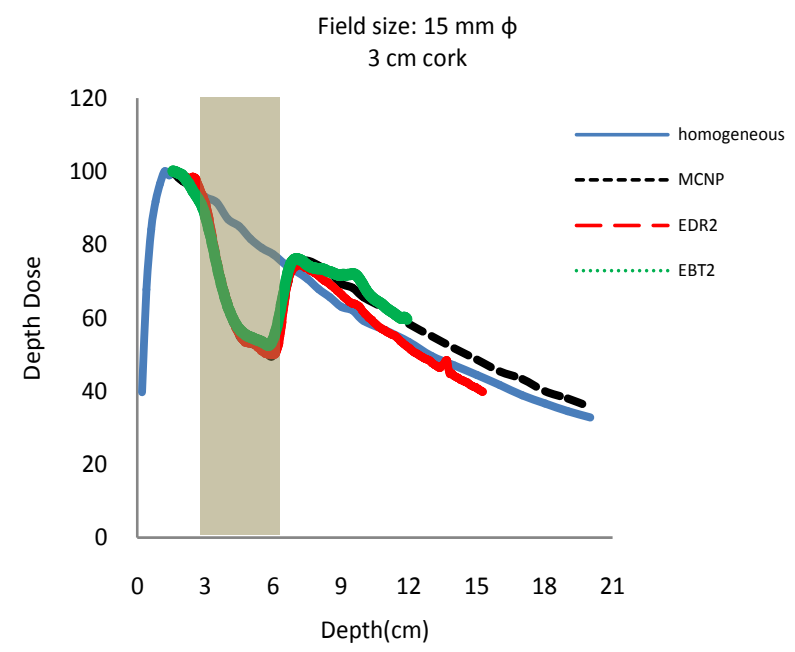

(c)

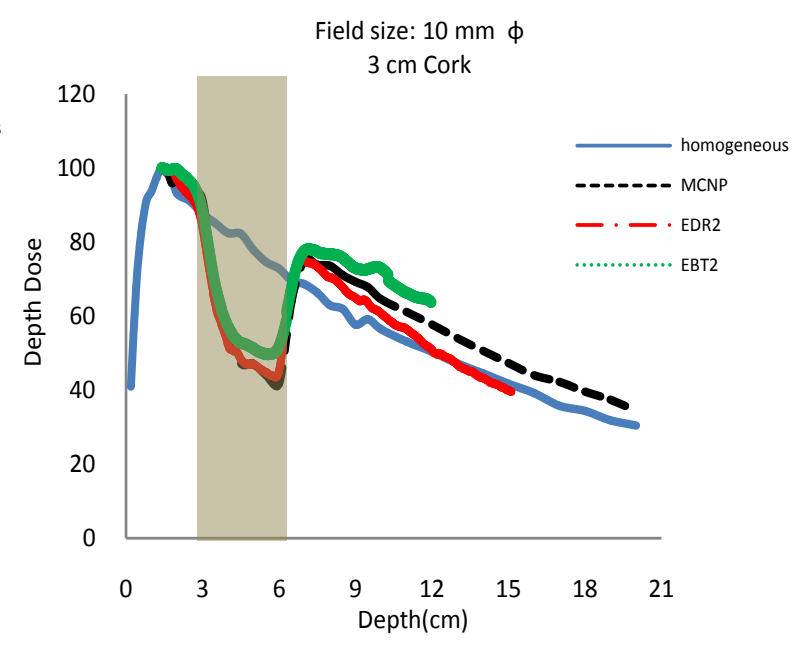

(b)

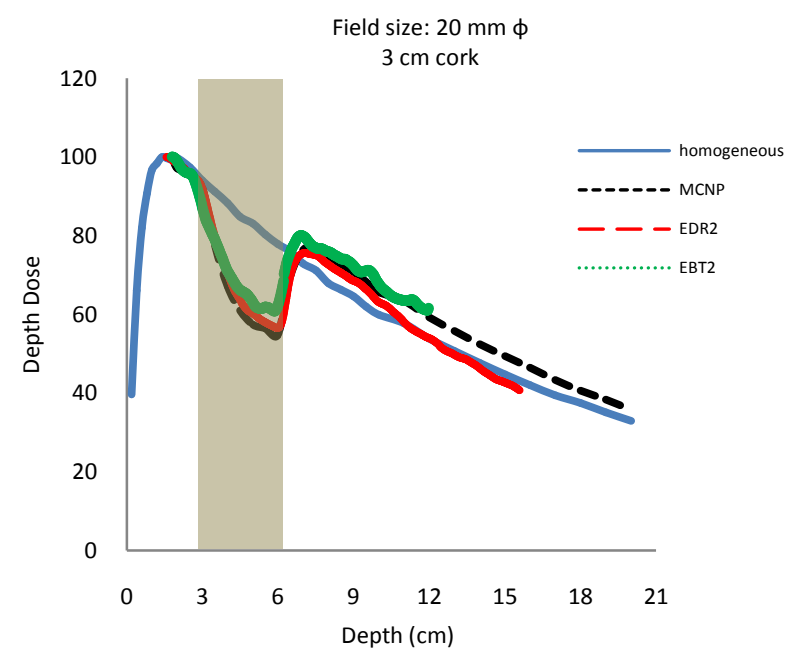

(d)

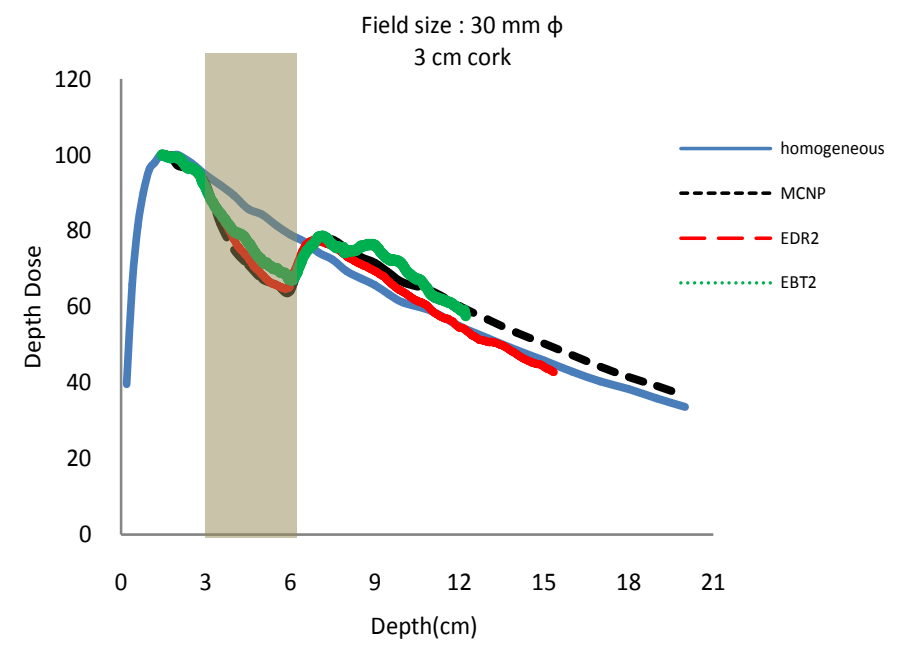

(e)

Figure 3. Measured and calculated PDD in heterogeneous phantom with $3 \mathrm{~cm}$ of cork (low density) that was irradiated in (a) 5, (b) 10, (c) 15, (d) 20 and (e) 30 mm field sizes. 


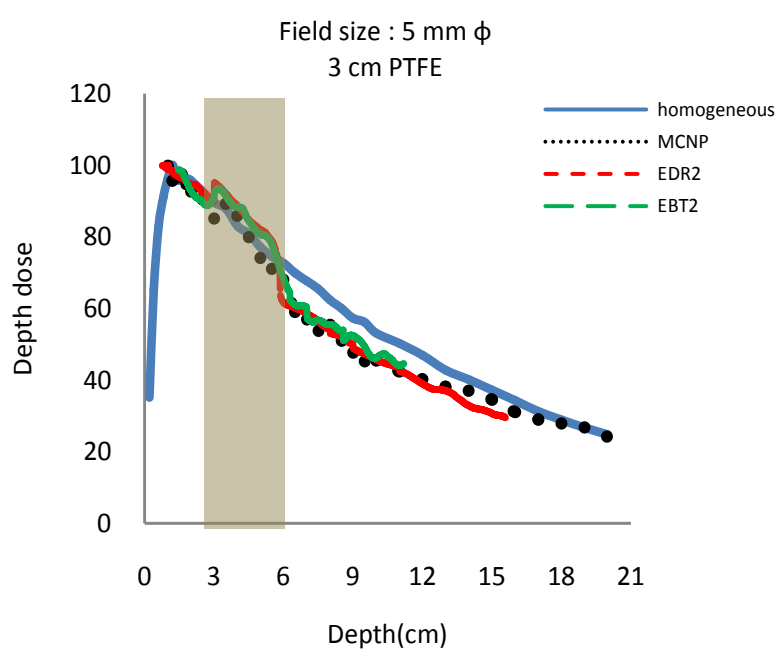

(a)

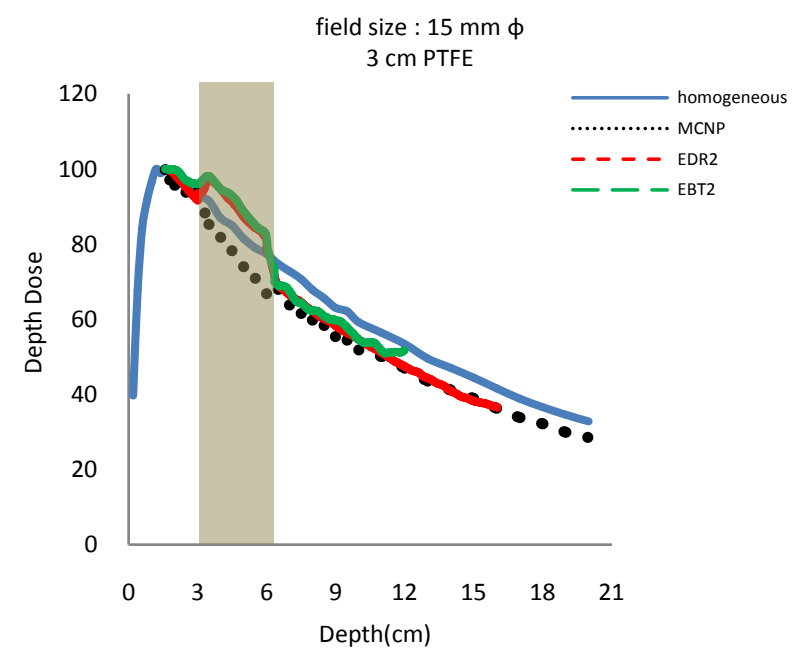

(c)

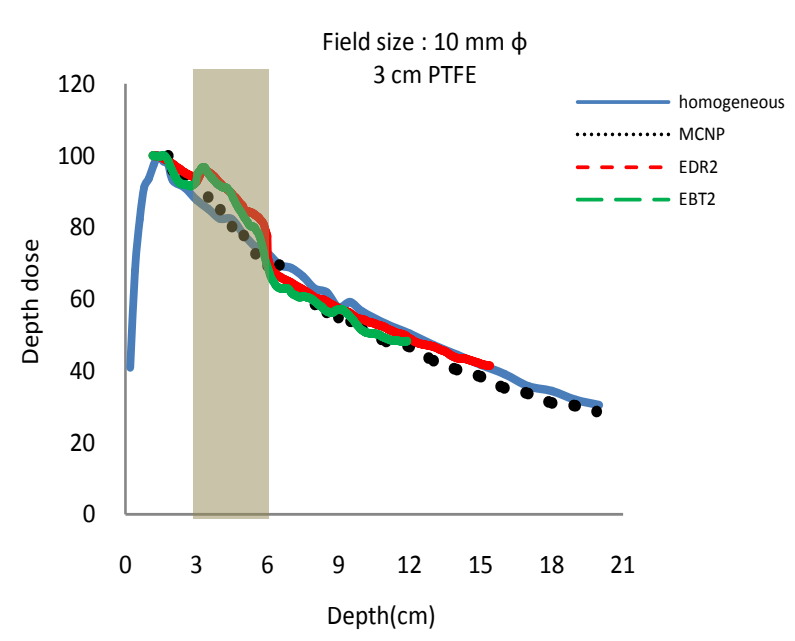

(b)

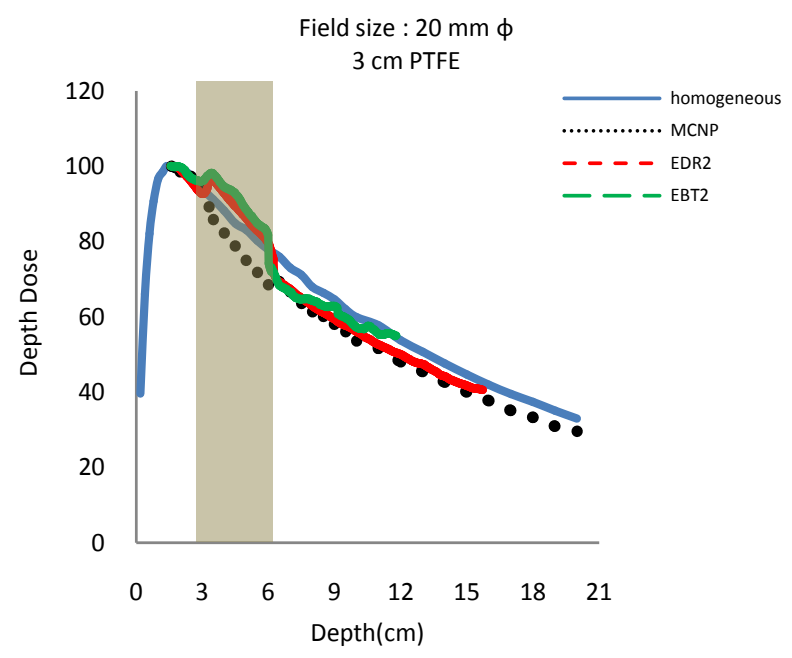

(d)

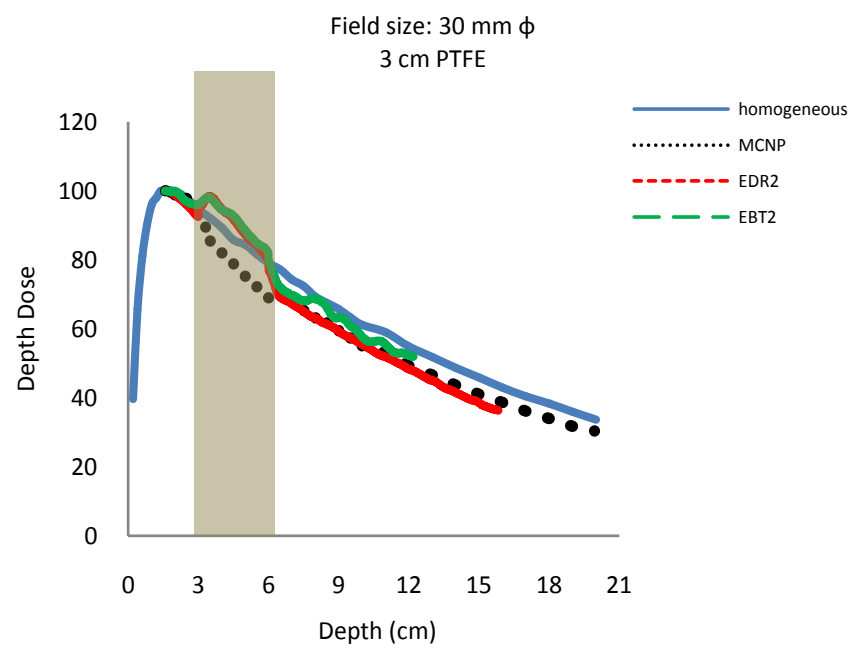

(e)

Figure 4. Measured and calculated PDD in heterogeneous phantom with $3 \mathrm{~cm}$ of P.T.F.E (high density) which was irradiated with 5, 10, 15, 20 and $30 \mathrm{~mm}$ diameter circular beams. 


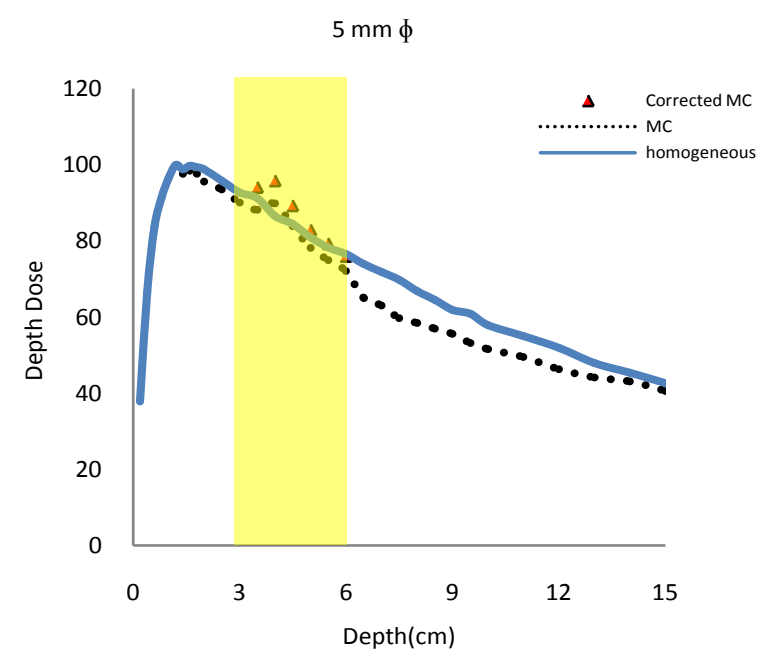

(a)

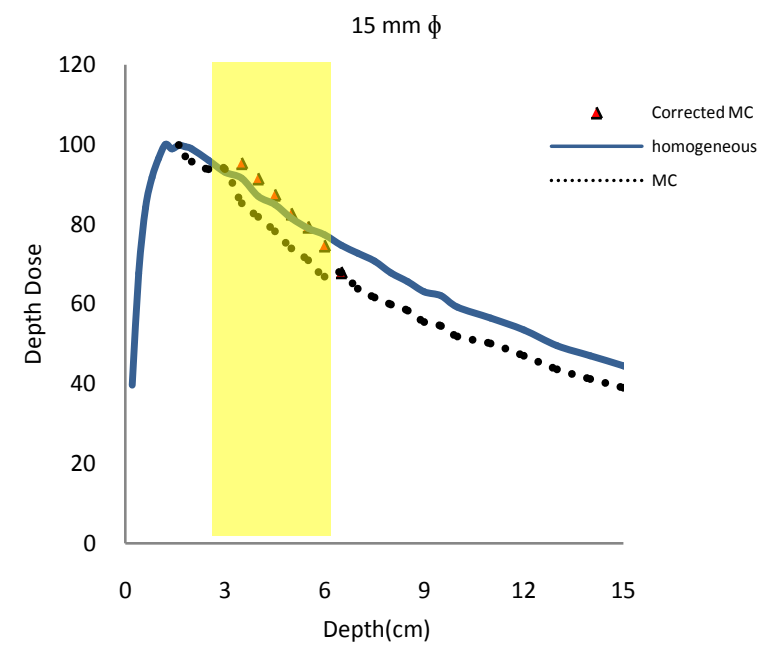

(c)

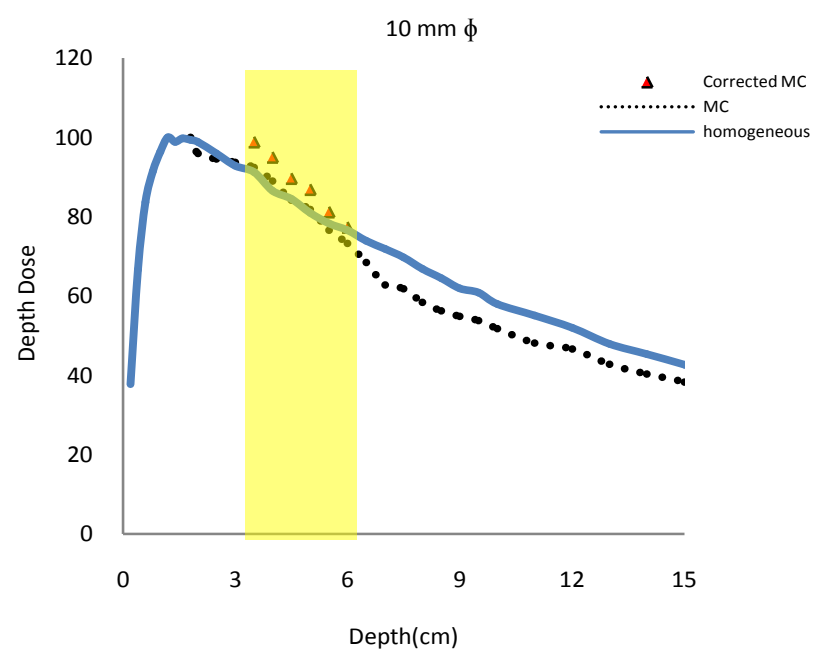

(b)

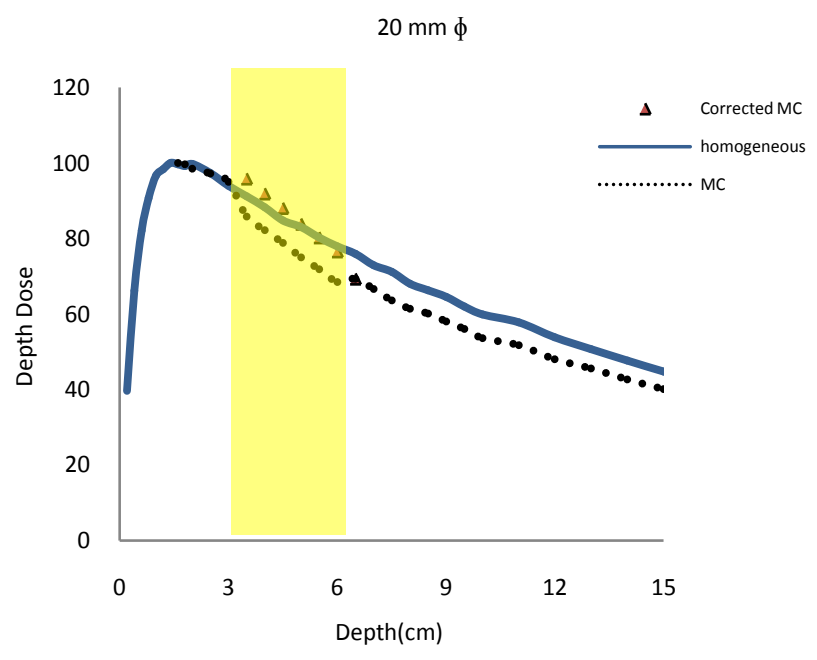

(d)

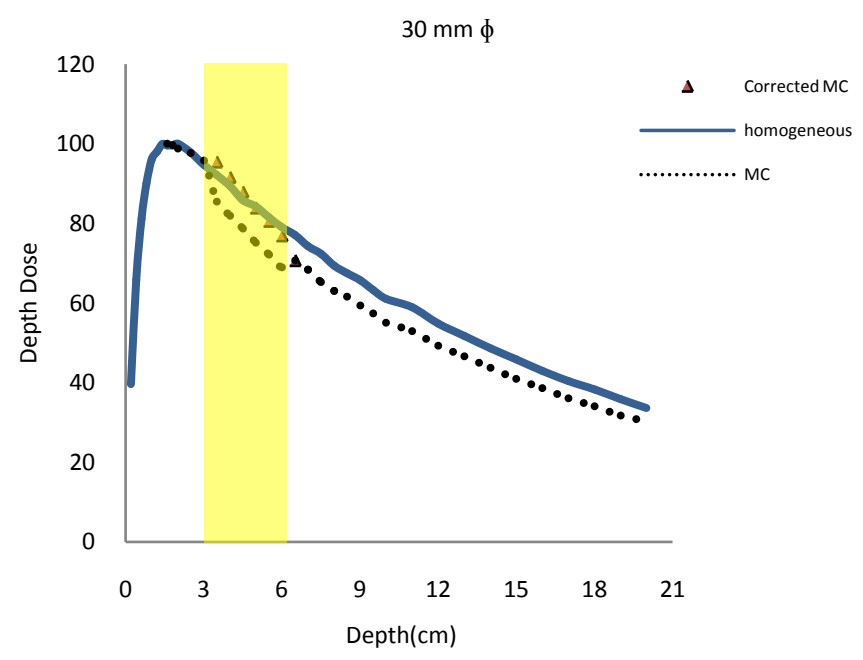

(e)

Figure 5. Calculated PDD in heterogeneous phantom before and after correction with $3 \mathrm{~cm}$ of P.T.F.E (high density) that irradiated with 5, 10, 15, 20 and $30 \mathrm{~mm}$ diameter circular beams. 
Table 1. Maximum dose variations inside the cork relative to homogeneous phantom.

\begin{tabular}{cccc}
\hline Field size $(\mathrm{mm})$ & EDR2 $(\%)$ & EBT2 $(\%)$ & MCNP (\%) \\
\hline 5 & 43.35 & 35.76 & 40.1 \\
10 & 36.75 & 32.45 & 37.2 \\
15 & 31.26 & 28.14 & 30 \\
20 & 23.56 & 20.82 & 25.7 \\
30 & 16.40 & 13.35 & 17 \\
\hline
\end{tabular}

Table 2. Maximum dose variations inside the P.T.F.E relative to homogeneous phantom.

\begin{tabular}{ccccc}
\hline Field size & EDR2 (\%) & EBT2 (\%) & MC (\%) & Corrected MC (\%) \\
\hline $5 \mathrm{~mm}$ & 7.16 & 6.12 & 4.74 & 7.3 \\
$10 \mathrm{~mm}$ & 10.79 & 11.66 & 3.27 & -6.19 \\
$15 \mathrm{~mm}$ & 5.9 & 6.65 & -10.02 & 5.71 \\
$20 \mathrm{~mm}$ & 5.27 & 6.9 & -6.7 & 5.59 \\
$30 \mathrm{~mm}$ & 6.34 & 6.1 & & 6.3 \\
\hline
\end{tabular}

deflected from multiple scattering and it is enhanced in higher atomic number materials like bone [20] which causes the change in energy and dose in central axis. Electron density in bone is smaller than soft tissue, therefore in Compton scattering area that depends to electron density, both EDR2 and EBT2 films show increase in dose.

Monte Carlo data show increasing of dose at first layer in central axis for 5, $10 \mathrm{~mm}$ collimator sizes because the effect of multiple scatterings on central axis is rule over and decreases the dose for 15, 20 and $30 \mathrm{~mm}$ collimator sizes. After high density heterogeneity, decrease of dose is observed because of shielding effect of bone. This effect is related to the thickness of bone and almost is independent of field size. Since base of Monte Carlo dose calculations typically report absorbed dose to the medium in which the particle transport took place [19], comparison of Dose-to-medium $(D m)$ with dose-to-water $(D w)$, require a conversion of $D m$ to $D w$. This conversion was made on the Bragg-Gray theory, $D w=D m(S w / S m)$. Therefore as it was depicted in Figure 5, if the results obtained by Monte Carlo code are multiply to water-to-medium mass collision stopping power over the energy spectra of primary electrons at the point of interest [19], MCNP data will appear near the data of the films.

\section{Conclusion}

Both EDR2 radiographic and EBT2 radiochromic films showed good agreement in low density medium as well as Monte Carlo Code. Results showed that dose variations in low density medium depend on density and field sizes that were used in this study (from 5 - $30 \mathrm{~mm}$ diameter). Inside the high density medium, the EDR2 and EBT2 results are similar but differ from MCNP data. After MCNP data correction using Bragg-Gray theory, both films and MCNP show good agreement with each other. Electron densities in high density medium and in Compton scattering area are important factors for variations and these variations are almost independent of field size.

\section{References}

[1] Podgorsak, B., Pike, G.B., Pla, M., Olivier, A. and Souhami, L. (1990) Radiotherapy and Oncology, 17, 349-358. http://dx.doi.org/10.1016/0167-8140(90)90008-K

[2] Wu, A., Zwicker, R.D., Kalend, A.M. and Zheng, Z. (1993) Medical Physics, $20,777$. http://dx.doi.org/10.1118/1.597032

[3] Bjärngard, B.E., Tsai, J.S. and Rice, R.K. (1990) Medical Physics, 17, 794. http://dx.doi.org/10.1118/1.596475 
[4] Rice, R.K., Hansen, J.L., Svensson, G.K. and Siddon, R.L. (1987) Physics in Medicine and Biology, 32, $1087-1099$. http://dx.doi.org/10.1088/0031-9155/32/9/002

[5] Rustgi, SN. and Frye, D.M. (1995) Medical Physics, 22, 2117-2121. http://dx.doi.org/10.1118/1.597655

[6] Westermark, M., Arndt, J., Nilsson, B. and Brahme, A. (2000) Physics in Medicine and Biology, 45, 685-702. http://dx.doi.org/10.1088/0031-9155/45/3/308

[7] Martens, C., De Wagter, C. and De Neve, W. (2000) Physics in Medicine and Biology, 45, 2519-2530. http://dx.doi.org/10.1088/0031-9155/45/9/306

[8] Verhaegen, F., Das, I.J. and Palmans, H. (1998) Physics in Medicine and Biology, 43, 2755-2768. http://dx.doi.org/10.1088/0031-9155/43/10/006

[9] Rustgi, S.N., Rustgi, A.K., Jiang, S.B. and Ayyangar, KM. (1998) Physics in Medicine and Biology, 43, 3509-3518. http://dx.doi.org/10.1088/0031-9155/43/12/009

[10] Ahn, Y.C., Lee, K.C., Kim, D.Y., Huh, S.J., Yeo, I.H., Lim, D.H., Kim, M.K., Shin, K.H., Park, S. and Chang, S.H. (2000) International Journal of Radiation Oncology • Biology • Physics, 48, 501-505. http://dx.doi.org/10.1016/S0360-3016(00)00612-X

[11] Cheung, J.Y., Yu, K.N., Yu, C.P. and Ho, R.T. (2001) Applied Radiation and Isotopes, 54, 461-465. http://dx.doi.org/10.1016/S0969-8043(00)00283-9

[12] Jones, A.O., Das, I.J. and Jones Jr., F.L. (2003) Medical Physics, 30, 296-300. http://dx.doi.org/10.1118/1.1539040

[13] Carrasco, P., Jornet, N., Duch, M.A., Panettieri, V., Weber, L., Eudaldo, T., Ginjaume, M. and Ribas, M. (2007) Medical Physics, 34, 3323-3333. http://dx.doi.org/10.1118/1.2750972

[14] Klein, E.E., Chin, L.M., Rice, R.K. and Mijnheer, B.J. (1993) International Journal of Radiation Oncology, Biology, Physics, 27, 419-427.

[15] Jones, A.O. and Das, I.J. (2005) Medical Physics, 32, 766-776. http://dx.doi.org/10.1118/1.1861154

[16] Pappas, E., Maris, T.G., Zacharopoulou, F., Papadakis, A., Manolopoulos, S., Green, S. and Wojnecki, C. (2008) Medical Physics, 35, 4640-4648. http://dx.doi.org/10.1118/1.2977829

[17] Dogan, N., Leybovich, L.B. and Sethi, A. (2002) Physics in Medicine and Biology, 47, 4121-4130. http://dx.doi.org/10.1088/0031-9155/47/22/314

[18] Shi, C., Papanikolaou, N., Yan, Y., Weng, X. and Jiang, H. (2006) Journal of Applied Clinical Medical Physics, 7, 1-8.

[19] Siebers, J.V., Keall, P.V., Nahum, A.E. and Mohan, R. (2000) Physics in Medicine and Biology, 45, 983-995. http://dx.doi.org/10.1088/0031-9155/45/4/313

[20] AAPM Report (2004) Task Group 65. Tissue Inhomogeneity Corrections for Megavoltage Photon Beams. The American Association of Physicists in Medicine, No. 85, Madison. 
Scientific Research Publishing (SCIRP) is one of the largest Open Access journal publishers. It is currently publishing more than 200 open access, online, peer-reviewed journals covering a wide range of academic disciplines. SCIRP serves the worldwide academic communities and contributes to the progress and application of science with its publication.

Other selected journals from SCIRP are listed as below. Submit your manuscript to us via either submit@scirp.org or Online Submission Portal.
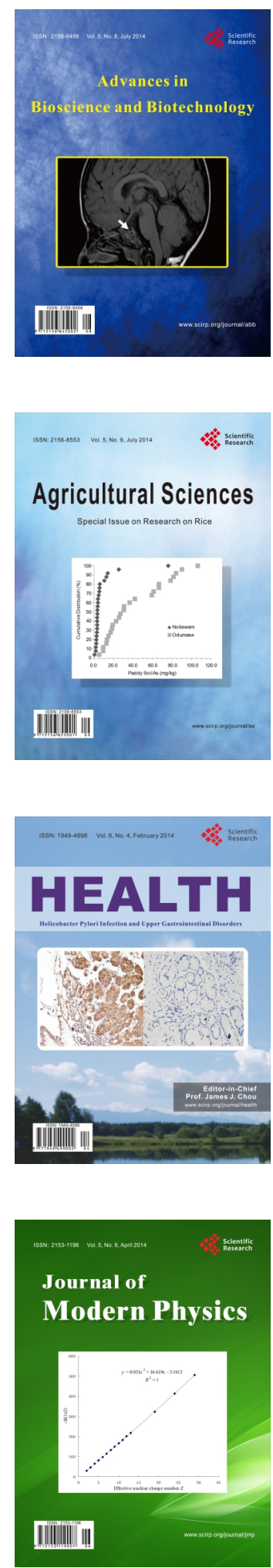
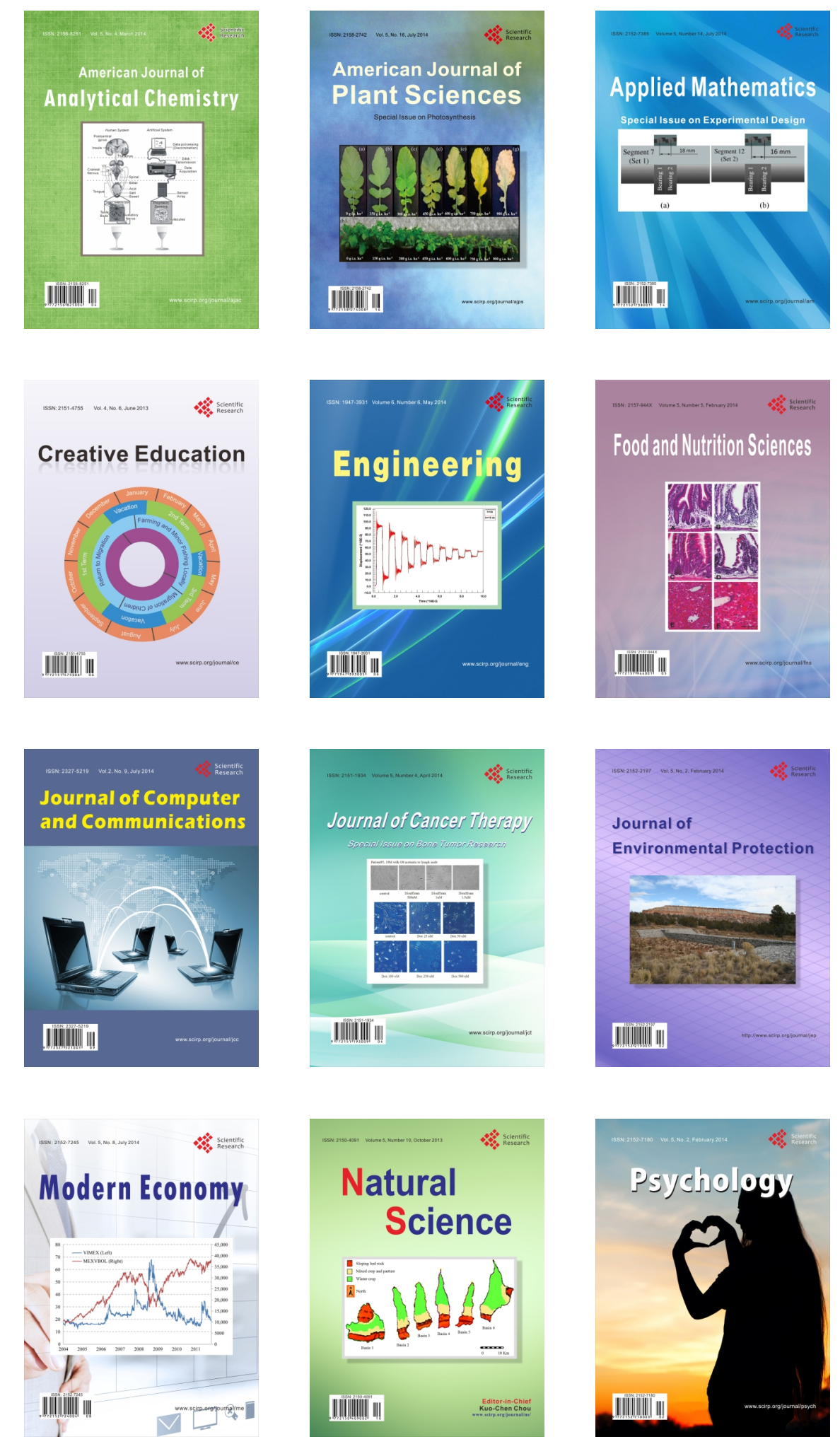